\title{
Special Topic: Novel Sensing Materials and Their Applications in Analytical Chemistry
}

\author{
Xi Chen ${ }^{1} \cdot$ Zhiyong Guo ${ }^{2}$
}

Published online: 18 March 2021

(c) The Nonferrous Metals Society of China 2021

Over the past years, the development and analytical applications of sensors and sensing materials which are pursued with great interest by many researchers have shown a tremendous potential growth. These studies and applications require interdisciplinary synergism in material, electrochemical and measurement methodological fronts. Compared with the classic materials, the novel sensing materials, especially nanomaterials, indicate more excellent characteristics and are widely applied for analytical sensing. With an insight to future trends in the ever growing fields of sensing materials, eight original papers are selected in this special issue to present the recent research achievements and the latest progress of noble sensing materials, as well as the developments of several typical novel sensing approaches. In this special issue, Lin et al., report a novel enzyme responded controlled release electrochemical biosensor, which utilized the enzyme to cleave the macromolecular HA-MB complex with enhanced electrochemical signal. Xia's group introduces monodisperse CdS supraparticles fluorescent tools for subcellular analysis, which show mitochondria targeting capacity and positively correlated with the length of the cationic surfactant molecules. Chen and her colleagues have prepared a $\mathrm{H}_{2} \mathrm{O}_{2}$ quenched sensing cysteine functionalized copper nanoclusters for hypoxanthine detection and reflecting the initial freshness of aquatic products. Yi et al., put forward a smartphone-based POCT system for the on-site quantitation of $\mathrm{Fe}^{3+}$, vitamin 12 and folate with effective interventions in the future. A multiresistant $\mathrm{SiO}_{2} @ \mathrm{CsPbX} \mathrm{Sb}_{3} @ \mathrm{SiO}_{2}$ perovskite composites have been prepared by Zhang's group, which demonstrate high sensing sensitivity towards $\mathrm{Fe}^{3+}$ in water sample. Zeng and his collaborators have developed a microfluidic paper analysis device and used the distances of color ribbons for distance-based detection of $\mathrm{Ag}^{+}$in water. Chen's group utilizes the halide exchange method and wavelength-shift characteristics of the $\mathrm{CsPbBr}_{3}$ PNCs to achieve the detection of chloridion in domestic water samples. In Rong's group, an accurate $\mathrm{NO}_{2}{ }^{-}$detection method has been established based on green fluorescent CDs which were prepared by one-pot hydrothermal carbonization and polymerization of acriflavine. An electrochemical flow device has been fabricated by Chen et al., for the real-time respond monitoring of $\mathrm{Cd}^{2+}$ and $\mathrm{Pb}^{2+}$ in fresh water samples.

Although several progresses for novel sensing materials are summarized and listed in this special issue for a broader readership including college students and academic researchers in analytical chemistry field, the topics and methodology presented in this issue are still limited due to the numbered papers. In the sensing applications or for sensor devices, sensitivity and the long-term stability are still great challenge, which demands the development of novel materials such as nano/MOF materials. In addition, more contributions focus on the development of sensing system such as micro-fluid analytical systems, and the innovative combination of physical principles and chemometrics applications will provide more help to increase the sensor characteristics. The editors would like to express their sincere appreciation to all authors for their efforts in preparing excellent contributions. Their efforts and cooperation enable the issue to be completed in time.
Xi Chen

xichen@xmu.edu.cn

$\triangle$ Zhiyong Guo

guozy@hxxy.edu.cn

1 Department of Chemistry, Xiamen University, Xiamen 361005, China

2 Xiamen Huaxia University, Xiamen 361024, China 


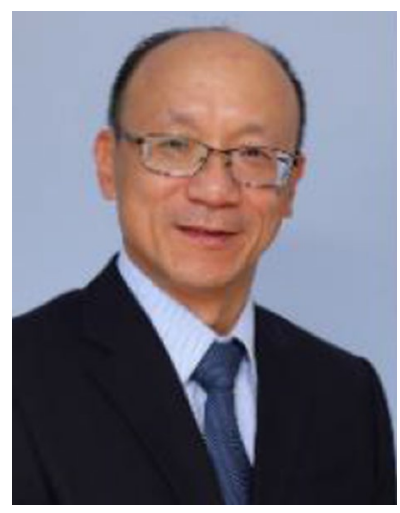

Xi Chen is a full Professor in the Department of Chemistry at Xiamen University, China. He received his Ph.D degree (1996) in Analytical Chemistry from the Kyoto Institute of Technology, Japan. Dr. Chen's research interests include electroanalytical chemistry, bio-chemical sensors and fluorescent nanomaterials. $\mathrm{He}$ has published over 305 scientific research articles in archival scientific journals.

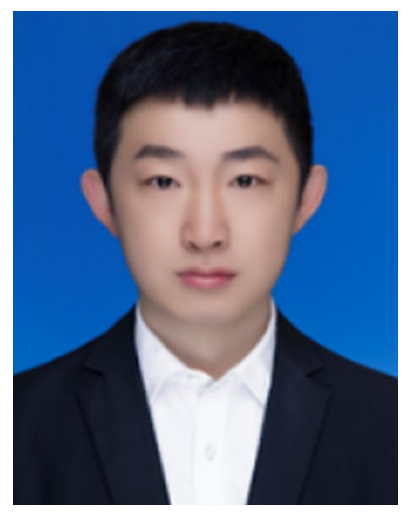

Zhiyong Guo is a lecturer in the Institute of Analytical Technology and Smart Instruments at Xiamen Huaxia University, China. He received his $\mathrm{Ph} . \mathrm{D}$ degree (2018) in Analytical Chemistry from the Northeastern University, China. Dr. Guo's interests include sample pretreatment, protein isolation and purification and novel nanomaterials construction. He has published over 10 scientific research articles in related scientific journals. 